\title{
RESEARCH
}

Open Access

\section{Identifying novel genetic variants for brain amyloid deposition: a genome-wide association study in the Korean population}

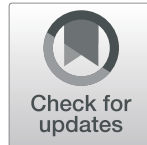

Hang-Rai Kim 1,2,3, Sang-Hyuk Jung 4,5, Jaeho Kim ${ }^{6}$, Hyemin Jang ${ }^{2,3}$, Sung Hoon Kang 2,3,7, Song Hwangbo 2,3, Jun Pyo Kim ${ }^{8}$, So Yeon Kim ${ }^{4,9}$, Beomsu Kim ${ }^{4}$, Soyeon Kim ${ }^{4}$, Jee Hyang Jeong ${ }^{10}$, Soo Jin Yoon ${ }^{11}$, Kyung Won Park ${ }^{12}$, Eun-Joo Kim ${ }^{13}$, Bora Yoon ${ }^{14}$, Jae-Won Jang ${ }^{15}$, Jin Yong Hong ${ }^{16}$, Seong Hye Choi ${ }^{17}$, Young Noh ${ }^{18}$, Ko Woon Kim ${ }^{19}$, Si Eun Kim ${ }^{20}$, Jin San Lee ${ }^{21}$, Na-Yeon Jung ${ }^{22}$, Juyoun Lee ${ }^{23}$, Byeong C. Kim² ${ }^{24}$ Sang Joon Son ${ }^{25}$, Chang Hyung Hong ${ }^{25}$, Duk L. Na ${ }^{2,3,26}$, Sang Won Seo $2,3,26,27$, Hong-Hee Won ${ }^{4,26^{*+}}$ and Hee Jin Kim ${\text { Ki, } 3,46^{*}+}_{\text {(D) }}$

\begin{abstract}
Background: Genome-wide association studies (GWAS) have identified a number of genetic variants for Alzheimer's disease (AD). However, most GWAS were conducted in individuals of European ancestry, and non-European populations are still underrepresented in genetic discovery efforts. Here, we performed GWAS to identify single nucleotide polymorphisms (SNPs) associated with amyloid $\beta$ (Aß) positivity using a large sample of Korean population.

Methods: One thousand four hundred seventy-four participants of Korean ancestry were recruited from multicenters in South Korea. Discovery dataset consisted of 1190 participants (383 with cognitively unimpaired [CU], 330 with amnestic mild cognitive impairment [aMCI], and 477 with AD dementia [ADD]) and replication dataset consisted of 284 participants (46 with $\mathrm{CU}, 167$ with aMCl, and 71 with ADD). GWAS was conducted to identify SNPs associated with $A \beta$ positivity (measured by amyloid positron emission tomography). A prediction models were developed using the identified SNPs. Furthermore, bioinformatics analysis was conducted for the identified SNPs.

Results: In addition to APOE, we identified nine SNPs on chromosome 7, which were associated with a decreased risk of A $\beta$ positivity at a genome-wide suggestive level. Of these nine SNPs, four novel SNPs (rs73375428, rs2903923, rs3828947, and rs1 1983537) were associated with a decreased risk of A positivity $(p<0.05)$ in the replication dataset. In a meta-analysis, two SNPs (rs7337542 and rs2903923) reached a genome-wide significant level $\left(p<5.0 \times 10^{-8}\right)$. Prediction performance for $A \beta$ positivity increased when rs 73375428 were incorporated (area under curve $=0.75 ; 95 \%$ $\mathrm{Cl}=0.74-0.76$ ) in addition to clinical factors and APOE genotype. Cis-eQTL analysis demonstrated that the rs73375428 was associated with decreased expression levels of FGL2 in the brain.
\end{abstract}

\footnotetext{
* Correspondence: wonhh@skku.edu; evekhj@gmail.com

${ }^{\dagger}$ Hong-Hee Won and Hee Jin Kim contributed equally to this work.

${ }^{4}$ Department of Digital Health, SAIHST, Sungkyunkwan University, Samsung

Medical Center, 81 Irwon-ro, Gangnam-gu, Seoul 06351, Republic of Korea

2Department of Neurology, Samsung Medical Center, Sungkyunkwan

University School of Medicine, 81 Irwon-ro, Gangnam-gu, Seoul 06351,

Republic of Korea

Full list of author information is available at the end of the article
}

(c) The Author(s). 2021 Open Access This article is licensed under a Creative Commons Attribution 4.0 International License, which permits use, sharing, adaptation, distribution and reproduction in any medium or format, as long as you give appropriate credit to the original author(s) and the source, provide a link to the Creative Commons licence, and indicate if changes were made. The images or other third party material in this article are included in the article's Creative Commons licence, unless indicated otherwise in a credit line to the material. If material is not included in the article's Creative Commons licence and your intended use is not permitted by statutory regulation or exceeds the permitted use, you will need to obtain permission directly from the copyright holder. To view a copy of this licence, visit http://creativecommons.org/licenses/by/4.0/ The Creative Commons Public Domain Dedication waiver (http://creativecommons.org/publicdomain/zero/1.0/) applies to the data made available in this article, unless otherwise stated in a credit line to the data. 
Conclusion: The novel genetic variants associated with $F G L 2$ decreased risk of $A \beta$ positivity in the Korean population. This finding may provide a candidate therapeutic target for AD, highlighting the importance of genetic studies in diverse populations.

Keywords: Alzheimer's disease, Amyloid-beta, Genome-wide association studies, Positron emission tomography

\section{Background}

Genetic factors play an important role in the pathogenesis of Alzheimer's disease (AD) because heritability is estimated to be $58 \%-79 \%$ [1]. In addition to APOE $\varepsilon 4$, recent genome-wide association studies (GWAS) have discovered a number of genetic risk variants for $\mathrm{AD}[2$, $3]$. However, a large proportion of AD heritability is still unexplained.

Accumulation of amyloid-beta $(A \beta)$ in the brain is the earliest pathogenic process in $\mathrm{AD}$, followed by tau deposition, neurodegeneration, and cognitive impairment [4]. Therefore, detecting individuals with $A \beta$ deposition is of utmost importance for the prevention and early treatment of AD [5]. Previous studies have evaluated the genetic basis of $A \beta$ deposition using positron emission tomography (PET) imaging [6-10] and identified several novel $\mathrm{A} \beta$ associated genetic variants outside the $A P O E$ region from European ancestry [11]. However, as each ancestry has a distinct genetic background, replication of the novel genetic findings in different populations is challenging. A number of previous studies failed to replicate European GWAS findings in other ethnic populations [12-15]. Furthermore, it should be noted that most previous GWAS were conducted in individuals of European ancestry, and non-European populations are underrepresented in genetic discovery efforts [16-18].

In this study, using a large sample of the Korean population, we conducted a GWAS to identify single nucleotide polymorphisms (SNPs) associated with $A \beta$ deposition in the brain. We identified novel SNPs for $A \beta$ deposition and demonstrated their associations in an independent cohort of the Korean population. Then, we assessed the topography of $A \beta$ deposition related to the novel SNP. Furthermore, we developed an $A \beta$ prediction model incorporating the novel SNP.

\section{Materials and methods Participants}

For the discovery dataset, total 1214 participants of Korean ancestry were recruited from 14 referral hospitals in South Korea from January 2013 to July 2019. Among them, 923 participants were recruited from the Samsung Medical Center, 201 participants were recruited from a multicenter study of the Korean Brain Aging Study for the Early Diagnosis and Prediction of AD (KBASE-V) [19], and 90 participants were recruited from a multicenter study of Clinical Research Platform based on Dementia Cohort.
For the replication dataset, we used data from 306 participants of Korean ancestry from the biobank of the Chronic Cerebrovascular Disease consortium, recruited from 2016 to 2018. This was part of the ongoing Biobank Innovation for chronic Cerebrovascular disease With ALZheimer's disease Study (BICWALZS) and the Center for Convergence Research of Neurological Disorders.

For the discovery and replication dataset, we included participants (i) who were diagnosed with amnestic mild cognitive impairment (aMCI), AD dementia (ADD), or were cognitively unimpaired $(\mathrm{CU})$ based on detailed neuropsychological tests [20-22], and (ii) who underwent amyloid PET imaging. Participants with aMCI met the following criteria, modified from Peterson's criteria [23]: (i) normal activities of daily living; (ii) objective memory impairment on verbal or visual memory test, below the 16th percentile of age- and educationmatched norms; and (iii) did not have dementia. Those with ADD satisfied the core clinical criteria for probable ADD according to the National Institute of Neurological and Communicative Disorders and Stroke and Alzheimer's Disease and Related Disorders Association [21]. We excluded participants if they had (i) a causative genetic mutation for AD, such as PSEN1, PSEN2, and APP; (ii) structural abnormalities detected on brain MRI, such as severe cerebral ischemia, territorial infarction, or brain tumors; and (iii) other medical or psychiatric diseases that may cause cognitive impairment. All participants provided written informed consent, and the study was approved by the Institutional Review Board of each center.

\section{Genotyping and imputation}

Participants were genotyped using the Illumina Asian Screening Array BeadChip (Illumina, CA, USA) for discovery data and Affymetrix customized Korean chips (Affymetrix, CA, USA) for replication data. Only SNP markers were analyzed. We conducted QC using PLINK software (version 1.9) [24]. Participants were excluded based on the following criteria: (i) call rate $<95 \%$, (ii) mismatch between reported and genetically inferred sex, (iii) deviation from each population parameter, (iv) excess heterozygosity rate (5 standard deviation from the mean), and (v) in cases of related pairs (identified with identity by descent $\geq 0.125$ ) within and between the discovery and replication datasets. 
SNPs were excluded based on the following criteria: (i) call rate $<98 \%$, (ii) minor allele frequency $(\mathrm{MAF})<1 \%$, and (iii) a $p$ value $<1.0 \times 10^{-6}$ for the Hardy-Weinberg equilibrium test. After $\mathrm{QC}$, genome-wide imputation was performed using the Minimac4 software with all available reference haplotypes from HRC-r1.1 on the University of Michigan Imputation Server [25, 26]. For post-imputation QC, we excluded SNPs based on the following criteria: (i) poor imputation quality $\left(\mathrm{r}^{2} \leq 0.8\right)$ and (ii) MAF $\leq 1 \%$. Finally, a total of 4,906,407 SNPs was analyzed.

\section{Amyloid PET acquisition and image analysis}

Amyloid PET images were obtained using a Discovery STE PET/CT scanner (GE Medical Systems, Milwaukee, WI, USA). PET images were acquired for $20 \mathrm{~min}$, starting at $90 \mathrm{~min}$ after intravenous injection of either ${ }^{18} \mathrm{~F}$ florbetaben or ${ }^{18} \mathrm{~F}$-flutemetamol. A $\beta$ positivity or negativity was determined by well-trained nuclear physicians using visual assessments for florbetaben and flutemetamol $[27,28]$ PET. Briefly, positivity for tracer uptake was assessed in four cortical regions (lateral temporal, frontal, parietal, and posterior cingulate cortices) for florbetaben PET and five cortical regions (lateral temporal, frontal, parietal, posterior cingulate cortices, and striatum) for flutemetamol PET. Amyloid PET positivity was defined as having at least one cortical region with evidence of positive uptake.

A subset of participants in the discovery cohort $(n=$ $824)$ and the replication cohort $(n=260)$ had amyloid PET data available for PET image analysis. For PET image analysis, we performed the following preprocessing using Statistical Parametric Mapping software 12 (SPM, http://www.fil.ion/uc.ac.uk/spm) running on MATLAB (MathWorks 2014b): (1) co-registration of PET to T1-weighted structural MRI, (2) structural MRI segmentation and calculation of transformation matrix, (3) normalization of PET to a Montreal Neurological Institute (MNI) space, and (4) spatial smoothing with a Gaussian kernel of 8-mm full width at half maximum. To calculate the standardized uptake value ratio (SUVR) for each PET image, we used two reference regions (the cerebellar cortex for florbetaben and pons for flutemeta$\mathrm{mol})$. The masks of reference regions were obtained from the GAAIN website (http://www.GAAIN.org).

\section{Statistical analysis GWAS analysis}

Logistic regression analysis was performed to determine the association between SNPs and A $\beta$ positivity controlling for age, sex, and the first three principal components (PC) of the genetic ancestry, expressed as $\mathrm{A} \beta$ positivity $=\beta_{0}+\beta_{1}$ age $+\beta_{2}$ sex $+\beta_{3} \mathrm{PC}_{1}+\beta_{4} \mathrm{PC}_{2}+\beta_{5}$ $\mathrm{PC}_{3}+\beta_{6}$ SNP (additive model, coded as 0,1 , and 2 according to the number of minor alleles). Reported $p$ values were two-tailed, and we defined a $p$ value less than $5.0 \times 10^{-8}$ as being statistically significant and less than $1.0 \times 10^{-5}$ or $1.0 \times 10^{-6}$ as being statistically suggestive based on previous studies [29-31]. We assessed genomic inflation according to a previous study [32]. For the replication analysis, reported $p$ values were twotailed, and a $p$ value less than 0.05 , was considered statistically significant. Furthermore, considering the small size of the replication dataset, we performed a permutation test to infer the statistical significance of SNPs from the null distribution. We recalculated the $t$ values of SNPs from logistic regression analysis of randomly shuffled $A \beta$ positivity (10,000 permutations). We calculated the fraction of permutations that showed a more significant association than the observed $t$ values of SNPs derived from the original dataset.

To check if SNPs were associated with $\mathrm{A} \beta$ positivity independent of $A P O E$ genotype, we performed a conditional analysis by further adjusting for $A P O E$ genotype. We also performed a $p$ value based meta-analysis and calculated the summary effect size by averaging the study specific effect sizes, with weights reflecting the standard errors from the study specific effect sizes.

\section{Effects of the newly identified SNPs}

After identifying associated SNPs, we calculated the risk of the identified SNPs on $A \beta$ deposition in all participants and at each cognitive level (CU, aMCI, and ADD). We also examined whether $A \beta$ associated SNPs are associated with ADD risk using $\mathrm{CU}$ and ADD participants using the following logistic model: ADD $=\beta_{0}+\beta_{1}$ age + $\beta_{2}$ sex $+\beta_{3}$ education $+\beta_{4}$ identified SNPs.

Next, using the previously reported cut-off values for $A \beta$ positivity (SUVR 0.6 for flutemetamol [33], and SUVR 1.4 for florbetaben [34]), we also performed logistic regression to evaluate whether the identified SNPs were associated with $A \beta$ deposition based on SUVR cutoff values.

Furthermore, we performed voxel-wise PET image analysis to determine which regional $\mathrm{A} \beta$ deposition is associated with SNPs after adjusting for the effects of age, sex, genetic PCs, APOE genotype, and PET tracer type. $\mathrm{T}$ static maps were thresholded by $p<0.001$ with cluster size $>20$ when uncorrected for multiple tests or $p<0.05$ when corrected for multiple tests using family-wise rate.

To test the clinical utility of the newly identified SNPs, we developed multivariable logistic models to predict $A \beta$ positivity in each individual. To evaluate the performance of the logistic model, we measured the area under curve (AUC) from the receiver operating characteristic curve analysis. For internal validation, we conducted a 10 -fold cross-validation with 100 repeats using the discovery data. We reported the mean AUC with 95\% 
confidence interval (CI) of the model. As an external validation, parameters estimated from the discovery data were used to test the $A \beta$ prediction performance in the replication data. We used $\mathrm{R}$ software (http://www.rproject.org) and MATLAB for the statistical analyses and results visualization.

Finally, we characterized the function of the identified SNPs by leveraging bioinformatic tools and previously reported results. First, we checked whether MAF of SNPs in our data was similar to that in the East Asian population using the 1000 Genomes Project dataset [35]. To evaluate the genotype-specific expression of identified SNPs in human brain tissues, we performed cisexpression quantitative trait loci (cis-eQTL) analysis through the Genotype-Tissue Expression portal (https:// gtexportal.org) [36]. We reported genes that showed significant expression changes in the brain tissues $(p<$ 0.05).

\section{Results}

\section{Participants}

After QC of genotype data, a total of 1190 (383 CU, 330 aMCI, and $477 \mathrm{ADD}$ ) and 284 participants (46 CU, 167 aMCI, and 71 ADD) remained available for the discovery and replication data, respectively. Table 1 shows the baseline demographics for the two datasets (discovery and replication data).

\section{GWAS analysis}

A quantile-quantile plot of $p$ values revealed no genomic inflation $(\lambda=1.008)$ (Fig. 1a). In the discovery data, we identified 61 genome-wide significant SNPs on chromosome $19\left(p<5.0 \times 10^{-8}\right)$ (Fig. 1b). However, all significant SNPs fell within the $500 \mathrm{~kb}$ region surrounding $A P O E$ and lost genome-wide significance when we adjusted for the $A P O E \& 4$ allele (Table S1). Outside of the $A P O E$ region, 38 SNPs on chromosomes $1,7,8,12$, and $22\left(p<1.0 \times 10^{-5}\right)$, and nine SNPs on chromosome $7(<$
$1.0 \times 10^{-6}$ ) showed genome-wide suggestive significance (Table S2). Among the nine SNPs, four were associated with $\mathrm{A} \beta$ positivity $(p<0.05)$ in the replication dataset (Table 2). The permutation test of all four SNPs showed t-values lower than the lowest $5 \%$ of 10,000 permutations (Table 2, Figure S1).

Of the four SNPs, rs11983537 was genotyped while the remaining were imputed. Imputation qualities of the identified SNPs were high (mean $r^{2} 0.97 \pm 0.02$ ). Of note, two of the four SNPs (rs73375428 and rs2903923) showed genome-wide significant associations $(p<5.0 \times$ $10^{-8}$ ) in the meta-analysis of the discovery and replication datasets (Table 2). When we adjusted for the effect of the APOE \&4 allele, all four SNPs were associated with $\mathrm{A} \beta$ positivity in the replication datasets $(p<0.05)$ (Table 2 ). Since the identified four SNPs showed high linkage disequilibrium (mean $r^{2} 0.95 \pm 0.05$ ) with each other, we selected rs73375428 for subsequent analyses because it showed the most significant association in the primary analysis of the discovery dataset.

\section{Effects of the newly identified SNPs}

In the logistic model, the $A P O E \varepsilon 4$ allele was associated with a 5 -fold higher risk of $A \beta$ positivity (odds ratio [OR] $=5.330 ; 95 \% \mathrm{CI}=4.188-6.788 ; p<0.001$ ) and rs73375428 was associated with a 2 -fold lower risk of $A \beta$ positivity $(\mathrm{OR}=0.519 ; 95 \% \mathrm{CI}=0.404-0.666 ; p<0.001)$. When we adjusted the effect of diagnosis (CU, aMCI, and ADD), the effect of rs73375428 remained significant $(\mathrm{OR}=0.556$; $95 \% \mathrm{CI}=0.406-0.666 ; p<0.001)$. In the subgroup analysis, the association of rs73375428 with $A \beta$ positivity was significant in the $\mathrm{CU}$ and aMCI groups but not in the ADD group, while the association of $A P O E \varepsilon 4$ was significant across all cognitive states (Table 3 ). When we defined $A \beta$ positivity based on SUVR, rs73375428 was also associated with a decreased risk of $A \beta$ positivity in both discovery $(\mathrm{OR}=0.608 ; 95 \% \mathrm{CI}=0.523-0.707 ; p<0.001)$ and

Table 1 Demographics of study participants

\begin{tabular}{|c|c|c|c|c|c|c|c|c|c|}
\hline \multirow[b]{2}{*}{ Demographics } & \multicolumn{4}{|c|}{ Discovery data } & \multicolumn{4}{|c|}{ Replication data } & \multirow[b]{2}{*}{$p^{t+}$} \\
\hline & $\begin{array}{l}\text { Total } \\
(n=1190)\end{array}$ & $\begin{array}{l}A \beta \text { negative } \\
(n=561)\end{array}$ & $\begin{array}{l}\text { A } \beta \text { positive } \\
(n=629)\end{array}$ & $p \dagger$ & $\begin{array}{l}\text { Total } \\
(n=284)\end{array}$ & $\begin{array}{l}A \beta \text { negative } \\
(n=180)\end{array}$ & $\begin{array}{l}\text { A } \beta \text { positive } \\
(n=104)\end{array}$ & $p^{\dagger}$ & \\
\hline Age, year (SD) & 70.07 (8.75) & $70.06(8.16)$ & 70.07 (9.25) & 0.990 & $72.67(7.32)$ & $71.76(7.31)$ & 74.25 (7.10) & 0.006 & $<0.001$ \\
\hline Female, n (\%) & $680(57.1)$ & $310(55.3)$ & $370(58.8)$ & 0.215 & $184(64.8)$ & $122(67.8)$ & $62(59.6)$ & 0.165 & 0.019 \\
\hline Education, year (SD) & $11.02(4.86)$ & $10.89(5.04)$ & $11.13(4.70)$ & 0.390 & $8.34(5.21)$ & $7.77(5.19)$ & $9.11(5.15)$ & 0.050 & $<0.001$ \\
\hline \multicolumn{10}{|l|}{ Diagnosis, n (\%) } \\
\hline $\mathrm{CU}$ & $383(32.2)$ & $326(58.1)$ & $57(9.1)$ & $<0.001$ & $46(16.2)$ & $43(23.9)$ & $3(2.9)$ & $<0.001$ & $<0.001$ \\
\hline $\mathrm{aMCl}$ & $330(27.7)$ & $172(30.7)$ & $158(25.1)$ & & 167 (58.8) & $125(69.4)$ & $42(40.4)$ & & \\
\hline ADD & $477(40.1)$ & $63(11.2)$ & $414(65.8)$ & & $71(25.0)$ & $12(6.7)$ & $59(56.7)$ & & \\
\hline
\end{tabular}

${ }^{\dagger} P$ value was calculated by comparing $A \beta$ negative and $A \beta$ positive participants. ${ }^{+\dagger} P$ value was calculated by comparing discovery data and replication data. Student's t test and chi-squared test were used for continuous and categorical variables, respectively.

Abbreviations: $A \beta$ amyloid $\beta, A D D$ Alzheimer's disease dementia, $a M C l$ amnestic mild cognitive impairment, $C U$ cognitive unimpaired, $S D$ standard deviation 
(A)
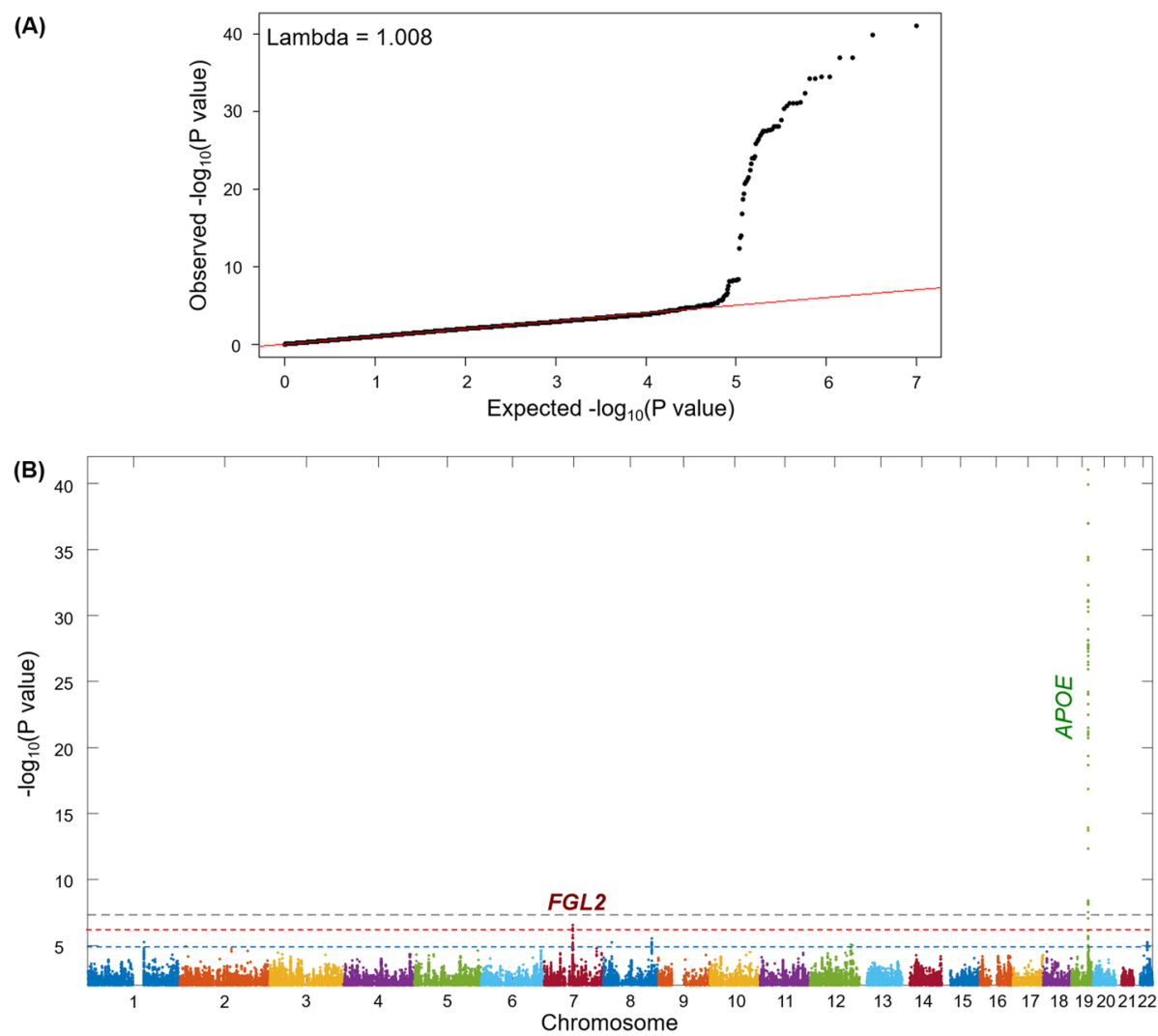

(C)

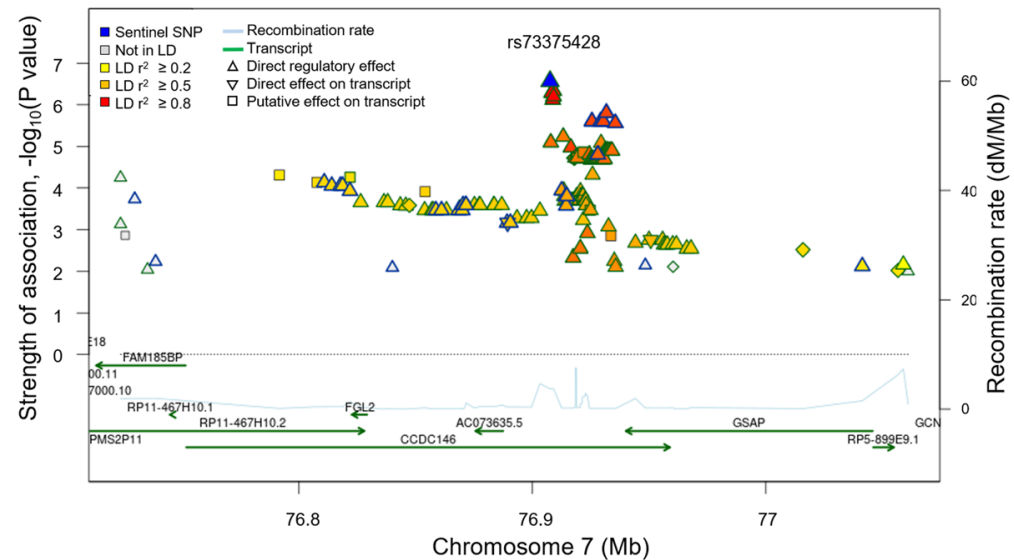

Fig. 1 a Q-Q plot. b Manhattan plot of GWAS analysis. c Regional association plot of rs73375428. The dotted line in the Manhattan plot indicate the genome-wide significance level $\left(p=5.0 \times 10^{-8}\right.$, gray dotted line) and the genome-wide suggestive level $\left(p=1.0 \times 10^{-6}\right.$, red dotted line; $p=$ $1.0 \times 10^{-5}$, blue dotted line), respectively. Regional association plot was modified from the SNiPA (single nucleotide polymorphism annotator) (https://snipa.helmholtz-muenchen.de/snipa3). GWAS, genome-wide association study; MAF, minor allele frequency; Q-Q plot, quantile-quantile plot; SNP, single nucleotide polymorphism; SPDYE18, speedy/RINGO cell cycle regulator family member E18; PMS2P9, PMS1 homolog2 mismatch repair system component pseudogene 9; FAM185BP, family with sequence similarity 185 member A pseudogene; SPDYE17, speedy/RINGO cell cycle regulator family member E17; 1-UPK3BP1-PMS2P11, uroplakin 3B pseudogene 1- PMS1 homolog2 mismatch repair system component pseudogene11; FGL2, fibrinogen-like protein 2; CCDC146, coiled-coil domain containing 146; GSAP, gamma secretase activating protein; GCNT1P5, glucosaminyl transferase 1 pseudogene 5

replication $(\mathrm{OR}=0.551 ; 95 \% \mathrm{CI}=0.408-0.744 ; p=0.047)$ datasets (Table S3).

In the voxel-wise PET image analysis, $A P O E \varepsilon 4$ was associated with increased $A \beta$ deposition on the wide cortical areas of the frontal, parietal, and temporal lobes. The SNP rs73375428 was associated with decreased $A \beta$ deposition in the precuneus, lateral parietal, and medial frontal areas, independent of age, 
Table 2 Associations between SNPs and A $\beta$ positivity in the two datasets

\begin{tabular}{|c|c|c|c|c|c|c|c|c|c|c|c|c|c|c|c|}
\hline \multirow[t]{3}{*}{ SNP } & \multirow[t]{3}{*}{ EA } & \multicolumn{7}{|c|}{ Analysis 1} & \multicolumn{7}{|c|}{ Analysis 2} \\
\hline & & \multicolumn{2}{|c|}{ Discovery data } & \multicolumn{3}{|c|}{ Replication data } & \multicolumn{2}{|c|}{ Meta-analysis } & \multicolumn{2}{|c|}{ Discovery data } & \multicolumn{3}{|c|}{ Replication data } & \multicolumn{2}{|c|}{ Meta-analysis } \\
\hline & & OR & $p$ & OR & $p$ & $p^{\dagger}$ & OR & $p$ & OR & $p$ & OR & $p$ & $p^{\dagger}$ & OR & $p$ \\
\hline rs73375428 & $G$ & 0.519 & $2.71 \times 10^{-7}$ & 0.550 & 0.040 & 0.0163 & 0.526 & $3.35 \times 10^{-8}$ & 0.535 & $1.23 \times 10^{-5}$ & 0.481 & 0.022 & 0.0101 & 0.516 & $8.00 \times 10^{-7}$ \\
\hline rs2903923 & G & 0.529 & $5.15 \times 10^{-7}$ & 0.539 & 0.032 & 0.0136 & 0.536 & $4.97 \times 10^{-8}$ & 0.546 & $2.19 \times 10^{-5}$ & 0.478 & 0.020 & 0.0058 & 0.510 & $1.32 \times 10^{-6}$ \\
\hline rs3828947 & C & 0.529 & $5.15 \times 10^{-7}$ & 0.547 & 0.036 & 0.0155 & 0.536 & $5.59 \times 10^{-8}$ & 0.546 & $2.19 \times 10^{-5}$ & 0.480 & 0.020 & 0.0056 & 0.515 & $1.39 \times 10^{-6}$ \\
\hline rs11983537 & T & 0.558 & $7.58 \times 10^{-7}$ & 0.539 & 0.026 & 0.0127 & 0.563 & $5.92 \times 10^{-8}$ & 0.570 & $1.99 \times 10^{-5}$ & 0.492 & 0.020 & 0.0091 & 0.517 & $1.22 \times 10^{-6}$ \\
\hline rs112599253 & T & 0.561 & $1.56 \times 10^{-7}$ & 0.723 & 0.214 & & & & 0.586 & $6.69 \times 10^{-5}$ & 0.698 & 0.210 & & & \\
\hline rs79761449 & $\mathrm{T}$ & 0.564 & $2.50 \times 10^{-7}$ & 0.723 & 0.214 & & & & 0.579 & $5.22 \times 10^{-5}$ & 0.698 & 0.210 & & & \\
\hline rs6971106 & T & 0.564 & $2.50 \times 10^{-7}$ & 0.723 & 0.214 & & & & 0.579 & $5.22 \times 10^{-5}$ & 0.698 & 0.210 & & & \\
\hline rs6978259 & $C$ & 0.522 & $4.62 \times 10^{-7}$ & 0.566 & 0.060 & & & & 0.521 & $7.90 \times 10^{-6}$ & 0.515 & 0.040 & & & \\
\hline rs6958464 & T & 0.526 & $6.28 \times 10^{-7}$ & 0.555 & 0.056 & & & & 0.524 & $9.63 \times 10^{-6}$ & 0.484 & 0.028 & & & \\
\hline
\end{tabular}

Analysis 1 is a logistic regression analysis, expressed as $A \beta$ positivity $=\beta_{0}+\beta_{1}$ age $+\beta_{2}$ sex $+\beta_{3} P C_{1}+\beta_{4} P C_{2}+\beta_{5} P C_{3}+\beta_{6} S N P$

Analysis 2 is a logistic regression analysis, expressed as $A \beta$ positivity $=\beta_{0}+\beta_{1}$ age $+\beta_{2}$ sex $+\beta_{3} P C_{1}+\beta_{4} P C_{2}+\beta_{5} P C_{3}+\beta_{6} A P O E \varepsilon 4+\beta_{7} S N P$

${ }^{\dagger} P$ values were calculated using permutation tests

Abbreviations: $B P$ base pair, $C$ cytosine, $C H R$ chromosome, $E A$ effective allele, $O R$ odds ratio, $G$ guanine, SNP single nucleotide polymorphism, $T$ thymine

sex, genetic PCs, $A P O E \varepsilon 4$, and PET tracer type (Fig. 2).

We additionally analyzed the risk of $A P O E \varepsilon 4$ and rs73375428 on the clinical diagnosis of ADD. APOE \&4 significantly increased $\mathrm{ADD}$ risk $(\mathrm{OR}=3.413 ; 95 \% \mathrm{CI}=$ 2.63-4.42; $p<0.001$ ) independent of age, sex, education, and rs73375428; and rs73375428 significantly decreased $\mathrm{ADD}$ risk $(\mathrm{OR}=0.579 ; 95 \% \mathrm{CI}=0.421-0.795 ; p<$ 0.001 ) independent of age, sex, education, and APOE \&4.

We developed prediction models to test the clinical utility of the $A P O E \& 4$ allele and newly identified SNP (rs73375428) in predicting $A \beta$ positivity. In the 10 -fold cross-validation with 100 repetitions, the model (model 1) including only clinical factors (age, sex, and level of education) showed an AUC of 0.506 (95\% CI $=0.500-0.512)$. After incorporating the $A P O E \& 4$ allele in the model (model 2), the prediction performance significantly increased $(\mathrm{AUC}=0.723 ; 95 \% \mathrm{CI}=0.717-0.729)$. Moreover, when the model included rs73375428 (model 3), the prediction performance further increased (AUC $=0.749 ; 95 \%$ $\mathrm{CI}=0.743-0.755$ ) (Fig. 3). When each model, trained in the discovery data, was tested in the replication data, the highest AUC was also observed in the model including both $A P O E$ \&4 and rs73375428 (model 1 AUC $=0.509$, model 2 AUC $=0.693$, model 3 AUC $=0.714)$.

\section{Cis-eQTL analysis}

rs73375428 was located in the intron of the coiled-coil domain containing the 146 (CCDC146) gene (Fig. 1c). After identifying three additional SNPs with high LD $\left(r^{2}\right.$ $>0.7$ ) (rs11983537, rs6978259, and rs3828947), we performed cis-eQTL analysis using the GTEx database. We found that two SNPs (rs73375428 and rs6978259) had significant cis-eQTL effects on the fibrinogen-like protein 2 (FGL2) gene in the brain cortex. Furthermore, a greater dosage of minor allele in SNPs was associated with decreased expression of FGL2 in the brain cortex (rs73375428, normalized effect size [NES] $=-0.175, p=$ 0.02 ; rs6978259, NES $=-0.176, p=0.01)$.

\section{Association of previously reported $A \beta$ risk loci from European populations with $A \beta$ positivity in the Korean population}

Among the $16 \mathrm{~A} \beta$-associated SNPs reported by Yan et al. [11], no SNP outside the $A P O E$ region showed significant association with $\mathrm{A} \beta$ positivity and only $M A G E F 1(\mathrm{OR}=$

Table 3 Risk of having a minor allele in rs73375428 (G) or APOE $\varepsilon 4$ on Aß positivity

\begin{tabular}{|c|c|c|c|c|}
\hline & \multicolumn{2}{|l|}{ rs73375428 } & \multicolumn{2}{|l|}{ APOE ع4 } \\
\hline & OR $(95 \% \mathrm{Cl})$ & $p$ & OR $(95 \% \mathrm{Cl})$ & $p$ \\
\hline Total $(n=1190)$ & $0.519(0.404-0.666)$ & $<0.001$ & $5.330(4.188-6.788)$ & $<0.001$ \\
\hline $\mathrm{CU}(n=383)$ & $0.486(0.244-0.964)$ & 0.030 & $3.885(2.307-6.54)$ & $<0.001$ \\
\hline $\mathrm{aMCl}(n=330)$ & $0.463(0.286-0.749)$ & 0.001 & $6.655(4.101-10.8)$ & $<0.001$ \\
\hline $\operatorname{ADD}(n=477)$ & $0.685(0.370-1.270)$ & 0.230 & $4.272(2.428-7.516)$ & $<0.001$ \\
\hline
\end{tabular}

Logistic regression analysis was adjusted for age and sex

Abbreviations: $A D D$ Alzheimer's disease dementia, $a M C l$ amnestic mild cognitive impairment, $C U$ cognitive unimpaired, $O R$ odds ratio 


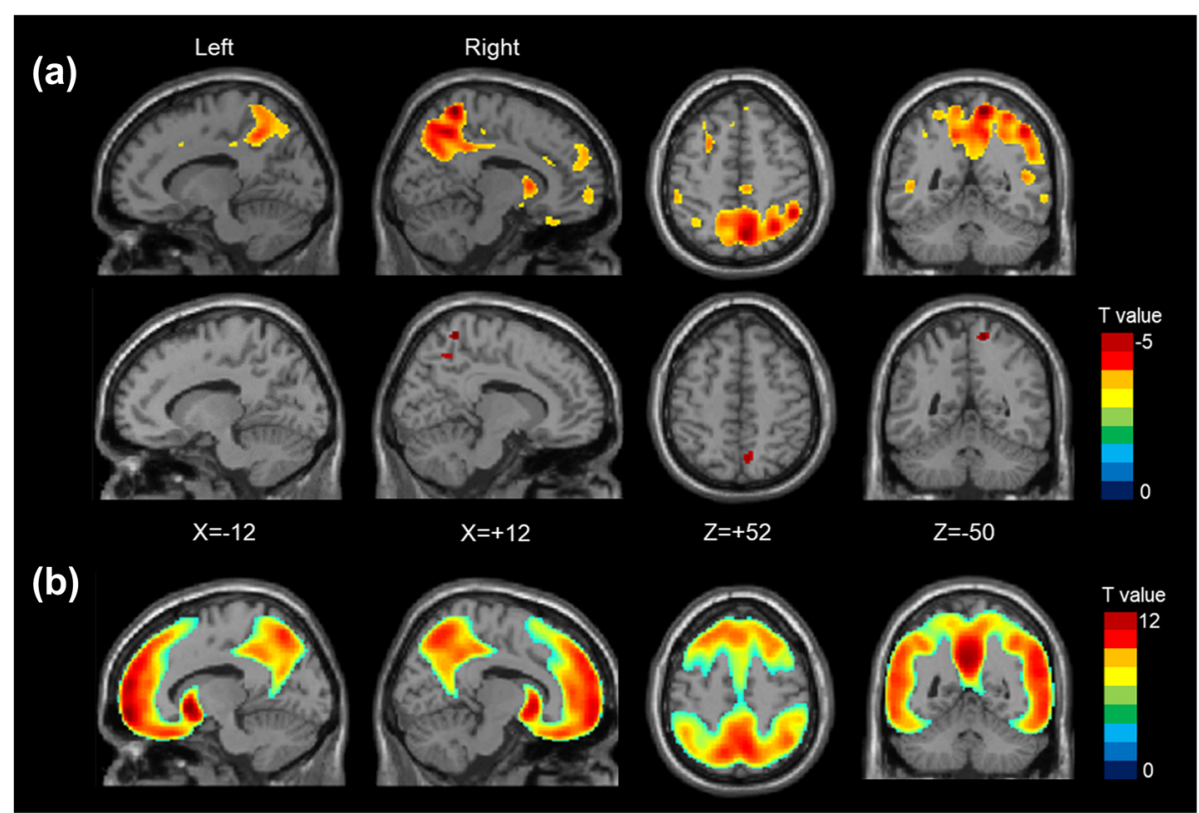

Fig. 2 Results of voxel-wise PET image analysis. T static maps showing a decreased A $\beta$ deposition in participants with the minor allele of the rs73375428 variant (first row: thresholded by uncorrected $p<0.001$ with cluster size $>20$; second row: thresholded by family-wise rate-corrected $p<0.05)$ and $\mathbf{b}$ increased $A \beta$ deposition in participants with APOE $\varepsilon 4$ allele (thresholded by family-wise rate corrected $p<0.05)$ ) $X$ and $Z$ are based on MNI coordinates. A $\beta$, amyloid $\beta$; MNI, Montreal Neurological Institute

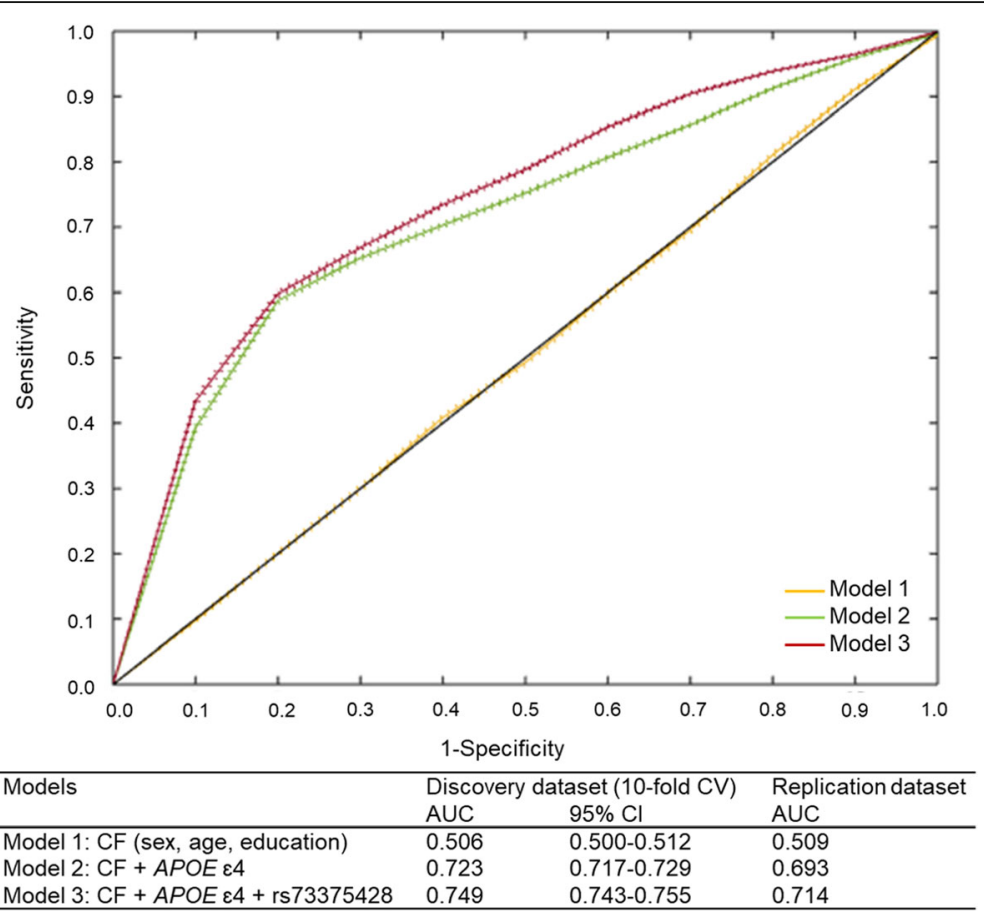

Fig. 3 ROC curves for the prediction of A positivity. Solid lines indicate the mean of AUC and dotted lines indicate 95\% Cls of AUC. Each model is developed by the multivariate logistic regression. $A \beta$, amyloid $\beta$; $A \cup C$, area under curve; $C F$, clinical factors; ROC, receiver-operating characteristic 
$0.810, p=0.058)$ locus showed marginal association in our cohort (Table S4). Based on the public dataset $(1000 \mathrm{Ge}-$ nomes Project phase 3) [35], the frequency of the previously reported SNPs differed between Europeans and East Asians, while our cohort (Korean) showed similar allele frequencies to that of East Asians (Table S4).

\section{Discussion}

We performed GWAS to identify genetic factors associated with $A \beta$ deposition in the brain using the largest amyloid PET imaging and GWAS data collected from multicenters in South Korea. We identified four novel SNPs (rs73375428, rs2903923, rs3828947, and rs11983537) on chromosome 7, which were associated with a decreased risk of $A \beta$ positivity in the brain at the suggestive level $\left(<1.0 \times 10^{-6}\right)$. These associations were also observed in the independent cohort $(p<0.05)$. Having a minor allele in rs73375428 (G) was associated with a 2 -fold decreased risk of $A \beta$ positivity $(O R=0.519)$ and decreased $A \beta$ deposition in the precuneus, lateral parietal, and medial frontal areas. Incorporating rs73375428, in addition to age, sex, education, and $A P O E$ e4, better predicted $\mathrm{A} \beta$ positivity. The minor allele of rs73375428 was associated with decreased expression levels of FGL2 in the brain.

We identified four novel SNPs (rs73375428, rs2903923, rs3828947, and rs11983537) associated with a decreased risk of $A \beta$ positivity in the brain. In the discovery dataset, nine SNPs showed genome-wide suggestive significance $\left(<1.0 \times 10^{-6}\right)$, of which four SNPs were associated with a decreased risk of $\mathrm{A} \beta$ positivity $(p<$ 0.05 ) in an independent cohort. Although the significance of four novel SNPs was at the suggestive level, meta-analysis of the discovery and replication datasets showed that two SNPs (rs73375428 and rs2903923) reached a genome-wide significance level $(p<5.0 \times$ $10^{-8}$ ). Furthermore, the obtained OR of rs73375428 for $\mathrm{A} \beta$ positivity was 0.519 , which was strong compared with the ORs of previously reported $A \beta$ - or ADDassociated SNPs (A $\beta$-associated SNPs OR from 0.84 to 1.2 [13]). In our cohort, about $30 \%$ of $\mathrm{CU}$ participants carried one or more minor alleles in rs73375428 (MAF of 0.160 ). This is in accordance with the previously reported MAF of rs73375428 in the East Asian population (MAF of 0.131) [35], which indicates that the samples used in this study were not biased and may reflect the East Asian population. In the subgroup analysis, the identified SNP (rs73375428) decreased the risk of $\mathrm{A} \beta$ positivity in the $\mathrm{CU}$ and aMCI group but not in the ADD group. This finding may suggest that in the course of AD spectrum, the effect of rs73375428 diminishes in the dementia stage.

Further imaging analysis and prediction model for $A \beta$ positivity showed consistent results. PET image analysis showed that the participants with minor allele in rs73375428 had less $\mathrm{A} \beta$ deposition in the precuneus, lateral parietal, and medial frontal areas. These areas are part of the default mode network, typical regions where $A \beta$ deposits in $A D$ [37]. Identifying patients with $A \beta$ deposition is of the utmost importance in predicting the prognosis and selecting patients for clinical trials of antiA $\beta$ therapy [38]. Currently available diagnostic tools for measuring $A \beta$ are either invasive (cerebrospinal fluid examination) or expensive (PET), hampering their widespread application in clinical practice [39]. We demonstrated that genetic data (APOE \&4 and rs73375428) obtained from blood samples with clinical information could predict $\mathrm{A} \beta$ positivity with an AUC of 0.749 . Furthermore, we demonstrated that the prediction performance improved when rs73375428 was included in the model in addition to age, sex, and $A P O E \varepsilon 4$, suggesting the clinical utility of rs73375428.

The identified SNPs were associated with decreased expression of FGL2 in the brain cortex. Although further specific biological mechanistic studies are required, this result suggests that $F G L 2$ may be a possible link between rs73375428 and decreased $A \beta$ deposition in the brain. FGL2 is a membrane-bound or secreted protein expressed by immune cells that have either coagulation activity [40,41] or immune-suppressive functions [42, 43]. A previous study demonstrated that FGL2 expression is associated with brain tumor progression through the immune system [44]. FGL2 was also associated with $\mathrm{AD}$. One prior study demonstrated that when human microglia were exposed to $\mathrm{A} \beta$ peptide, FGL2 expression in microglia was reduced more than six-fold as an inflammatory response to $A \beta$ peptide [45]. Furthermore, Taguchi et al. obtained brain samples from both patients with AD and controls of Japanese population and demonstrated that FGL2 was upregulated in the AD hippocampus as compared to controls [46]. Given these previous observations, we speculated that participants with minor alleles of rs73375428 could have reduced the risk of $A \beta$ deposition in the brain through decreased expression of $F G L 2$, which reflects the reactive inflammatory response (e.g., $A \beta$ clearance) to $A \beta$ peptide. More functional studies are necessary to elucidate the role of FGL2 in AD pathogenesis.

Our results showed some evidence for ethnic similarity and differences in genetic variants associated with $A \beta$. As expected, variants in the $A P O E$ locus exhibited a significant association with $\mathrm{A} \beta$ deposition in the brain, confirming that the $A P O E$ variants are important risk factors for $\mathrm{AD}$ across various ethnicities [47]. However, there were some ethnic differences. We observed a stronger effect of the variant in APOE (rs429358) on $\mathrm{A} \beta$ positivity in the Korean population than that in the European population (Korean, OR $=5.275$; European, 
$\mathrm{OR}=1.197$ [11]). This is similar to the results in previous studies of the East Asian population, in which the effect of $A P O E \& 4$ on AD risk was stronger in Han Chinese [48] and Japanese [47] than in the European population. Furthermore, outside the $A P O E$ locus, previously reported A $\beta$ associated SNPs in European ancestry data were not replicated [11] in our cohort. Ethnic differences in the effect size and significance might be attributed to the differences in allele frequency and LD pattern across different populations [12]. Indeed, we observed heterogeneity in the allele frequency between the European and Korean cohorts (Table S4). Furthermore, epigenomic patterns, lifestyle, education attainment, and other non-genetic factors may also account for differences across populations. However, it should be noted that the lack of replication might also be a result of insufficient sample size of our cohort. Nevertheless, these findings suggest that the discovery from GWAS in one population may not be applicable to other populations. Therefore, continuous efforts of population-specific and trans-ethnic studies are necessary to accurately discover risk genetic variants.

\section{Limitations}

This study has several limitations. First, the statistical significance of the novel SNP was at the genome-wide suggestive level, and the sample size of the replication dataset was small. Furthermore, although associations between four SNPs and $A \beta(p<0.05)$ were found in the independent dataset, the statistical significance disappeared after correction for multiple tests of nine SNPs. However, our study might present true findings for the following reasons: (i) nine suggestive SNPs at a more conservative $p$-value $\left(<1.0 \times 10^{-6}\right)$ showed high LD with each other, which might reduce the number of independent tests to one; (ii) the permutation test of the four SNPs showed that if the null hypothesis was true, the chance of observing our findings would be extremely small for a given sample size; (iii) two SNPs (rs73375428 and rs2903923) showed genome-wide significant associations in the meta-analysis; and (iv) the biological relevance of FGL2 association with the identified SNPs in the brain tissue suggests a potential $\mathrm{AD}$-associated gene. Nevertheless, our findings should be interpreted with caution and replicated in larger independent datasets. Second, imputation was performed using a large reference panel of mixed populations rather than the Korean population. However, we conducted a strict postimputation QC, excluding SNPs with poor imputation quality $\left(r^{2} \leq 0.8\right)$ or low frequency (MAF $\left.<1 \%\right)$. As a result, the imputation qualities of the identified SNPs were high (mean $r^{2} 0.97 \pm 0.02$ ). Third, the cis-eQTL dataset was obtained from healthy populations and not from subjects with AD. Furthermore, the causality of the identified SNPs and FGL2 expression could not be evaluated in the current analysis. Functional studies using gene editing are necessary to determine the association between the identified SNPs and FGL2. Fourth, GWAS was conducted using $A \beta$ positivity, determined by the visual assessment not by quantitative A $\beta$ SUVR. Since this study was conducted using large data obtained from multiple cohorts, some data were not available for SUVR analysis. However, the visual assessment of $A \beta$ positivity has high correlations with histopathological findings of $\mathrm{A} \beta$ deposition in the brain $[49,50]$, and it is more widely used in the clinical practice.

\section{Conclusions}

We identified novel SNPs that reduce the risk of $A \beta$ deposition in the brain and suggested a possible role of FGL2 in AD pathogenesis. This finding may provide a candidate therapeutic target for AD, highlighting the importance of genetic studies in diverse populations.

\section{Abbreviations}

Aß: Amyloid-beta; AD: Alzheimer's disease; ADD: Alzheimer's disease dementia; aMCl: Amnestic mild cognitive impairment; AUC: Area under curve; BICWALZS: Biobank Innovation for chronic Cerebrovascular disease With ALZheimer's disease Study; CCDC146: Coiled-coil domain containing the 146; Cl: Confidence interval; cis-eQTL: Cis-expression quantitative trait loci; CU: Cognitive unimpaired; FGL2: Fibrinogen-like protein 2; GWAS: Genome-wide association studies; MAF: Minor allele frequency; MNI: Montreal Neurological Institute; NES: Normalized effect size; OR: Odds ratio; PC: Principal component; PET: Positron emission tomography; SNP: Single nucleotide polymorphism; KBASE-V: Korean Brain Aging Study for the Early Diagnosis and Prediction of AD

\section{Supplementary Information}

The online version contains supplementary material available at https://doi. org/10.1186/s13195-021-00854-z.

Additional file 1: Table S1. Significant ( $p$ value $<5.0 \times 10^{-8}$ ) SNPS associated with $A \beta$ positivity. Table S2. Suggestive SNPs associated with $A \beta$ positivity. Table S3. Association of genome-wide suggestive SNPS $\left(p<1.0 \times 10^{-6}\right)$ with A $\beta$ positivity based on SUVR. Table S4. Association of previously reported $A \beta$ risk loci from European populations with $A \beta$ positivity in the Korean population.

Additional file 2: Figure S1. Histogram of t-values obtained from the permutations. Red dotted lines indicate the lowest $5 \%$ of the 10,000 permutations. Red arrows indicate the observed t-value obtained from the original dataset.

\section{Acknowledgements}

Not applicable.

\section{Authors' contributions}

H.R.K., H.H.W., and H.J.K. contributed to the study design, data collection, data analysis, and drafting the manuscript. S.H.J. contributed to the data collection, data analysis, and revising the manuscript. J.K., H.J., S.H.K., S.H., J.P.K., S.K., J.H.J., S.J.Y., K.W.P., E.J.K., B.Y., J.W.J., J.Y.H., S.H.C., Y.N., K.W.K., S.E.K., J.S.L., N.Y.J., Y.L., B.C.K., S.J.S., C.H.H., D.L.N., and S.W.S. contributed to the data collection, data interpretation, and revising the manuscript. The authors read and approved the final manuscript.

\section{Funding}

This research was supported by a National Research Foundation of Korea grant, funded by the Korean government (MSIP) (2018R1A1A3A04079255 
and 2014M3C7A1064752); the ICT Creative Consilience program (IITP-2020-001821) supervised by the Institute for Information \& Communications Technology Planning \& Evaluation (IITP); the Korea Health Technology R\&D Project through the Korea Health Industry Development Institute, funded by the Ministry of Health and Welfare, Republic of Korea (HU21C0111 and HI19C1132); the Ministry of Science and ICT, Republic of Korea (2019RIA5A2026045); and the Korea Centers for Disease Control and Prevention (2018-ER6202-01 and 4845-303).

\section{Availability of data and materials}

The datasets used and analyzed during the current study are available from the corresponding author on reasonable request.

\section{Declarations}

\section{Ethics approval and consent to participate}

All participants provided written informed consent, and the study was approved by the Institutional Review Board at all participating institutions.

\section{Consent for publication}

Not applicable

\section{Competing interests}

The authors declare that they have no competing interests.

\section{Author details}

'Department of Neurology, Dongguk University Ilsan Hospital, Dongguk University College of Medicine, Goyang, Republic of Korea. ${ }^{2}$ Department of Neurology, Samsung Medical Center, Sungkyunkwan University School of Medicine, 81 Irwon-ro, Gangnam-gu, Seoul 06351, Republic of Korea. ${ }^{3}$ Alzheimer's Disease Convergence Research Center, Samsung Medical Center, Seoul, Republic of Korea. ${ }^{4}$ Department of Digital Health, SAIHST, Sungkyunkwan University, Samsung Medical Center, 81 Irwon-ro, Gangnam-gu, Seoul 06351, Republic of Korea. ${ }^{5}$ Department of Biostatistics, Epidemiology and Informatics, Perelman School of Medicine, University of Pennsylvania, Philadelphia, USA. ${ }^{6}$ Department of Neurology, Dongtan Sacred Heart Hospital, Hallym University College of Medicine, Hwaseong, Republic of Korea. ${ }^{7}$ Department of Neurology, Korea University Guro Hospital, Korea University College of Medicine, Seoul, Korea. ${ }^{8}$ Center for Neuroimaging, Radiology and Imaging Sciences, Indiana University School of Medicine, Indianapolis, IN, USA. 'Samsung Genome Institute, Samsung Medical Center, Seoul, Republic of Korea. ${ }^{10}$ Department of Neurology, Ewha Womans University Seoul Hospital, Ewha Womans University School of Medicine, Seoul, Republic of Korea. ${ }^{11}$ Department of Neurology, Eulji University Hospital, Eulji University School of Medicine, Daejeon, Republic of Korea. ${ }^{12}$ Department of Neurology, Dong-A University College of Medicine, Department of Translational Biomedical Sciences, Graduate School of Dong-A University, Busan, Republic of Korea. ${ }^{13}$ Department of Neurology, Pusan National University Hospital, Pusan National University School of Medicine and Medical Research Institute, Busan, Republic of Korea. ${ }^{14}$ Department of Neurology, Konyang University College of Medicine, Daejeon, Republic of Korea. ${ }^{15}$ Department of Neurology, Kangwon National University Hospital, Kangwon National University College of Medicine, Chuncheon, Republic of Korea. ${ }^{16}$ Department of Neurology, Yonsei University Wonju College of Medicine, Wonju, Republic of Korea. ${ }^{17}$ Department of Neurology, Inha University School of Medicine, Incheon, Republic of Korea. ${ }^{18}$ Department of Neurology, Gachon University College of Medicine, Gil Medical Center, Incheon, Republic of Korea. ${ }^{19}$ Department of Neurology, School of Medicine, Jeonbuk National University Hospital, Jeonju, Republic of Korea. ${ }^{20}$ Department of Neurology, Inje University College of Medicine, Haeundae Paik Hospital, Busan, Republic of Korea. ${ }^{21}$ Department of Neurology, Kyung Hee University College of Medicine, Kyung Hee University Hospital, Seoul, Republic of Korea. ${ }^{22}$ Department of Neurology, Pusan National University Yangsan Hospital, Pusan National University School of Medicine and Medical Research Institute, Busan, Republic of Korea.

${ }^{23}$ Department of Neurology, Chungnam National University Hospital, Daejeon, Republic of Korea. ${ }^{24}$ Departmet of Neurology, Chonnam National University School of Medicine, Gwangju, Republic of Korea. ${ }^{25}$ Department of Psychiatry, Ajou University School of Medicine, Suwon, Republic of Korea.

${ }^{26}$ Department of Health Sciences and Technology, SAIHST, Sungkyunkwan
University, Seoul, Republic of Korea. ${ }^{27}$ Department of Intelligent Precision Healthcare Convergence, Sungkyunkwan University, Seoul, Republic of Korea.

Received: 20 February 2021 Accepted: 2 June 2021

Published online: 21 June 2021

\section{References}

1. Gatz M, Reynolds CA, Fratiglioni L, Johansson B, Mortimer JA, Berg S, et al. Role of genes and environments for explaining Alzheimer disease. Arch Gen Psychiatry. 2006;63(2):168-74. https://doi.org/10.1001/archpsyc.63.2.168.

2. Kunkle BW, Grenier-Boley B, Sims R, et al. Genetic meta-analysis of diagnosed Alzheimer's disease identifies new risk loci and implicates $A \beta$, tau, immunity and lipid processing. Nat Genet. 2019;51(3):414-30. https:// doi.org/10.1038/s41588-019-0358-2.

3. Jansen IE, Savage JE, Watanabe K, Bryois J, Williams DM, Steinberg S, et al. Genome-wide meta-analysis identifies new loci and functional pathways influencing Alzheimer's disease risk. Nat Genet. 2019;51(3):404-13. https:// doi.org/10.1038/s41588-018-0311-9.

4. Jack CR Jr, Knopman DS, Jagust WJ, Petersen RC, Weiner MW, Aisen PS, et al. Tracking pathophysiological processes in Alzheimer's disease: an updated hypothetical model of dynamic biomarkers. Lancet Neurol. 2013; 12(2):207-16. https://doi.org/10.1016/S1474-4422(12)70291-0.

5. Sperling RA, Aisen PS, Beckett LA, Bennett DA, Craft S, Fagan AM, et al. Toward defining the preclinical stages of Alzheimer's disease: recommendations from the National Institute on Aging-Alzheimer's Association workgroups on diagnostic guidelines for Alzheimer's disease. Alzheimers Dement. 2011;7(3): 280-92. https://doi.org/10.1016/j.jalz.2011.03.003.

6. Apostolova LG, Risacher SL, Duran T, Stage EC, Goukasian N, West JD, et al. Associations of the top 20 Alzheimer disease risk variants with brain amyloidosis. JAMA Neurol. 2018;75(3):328-41. https://doi.org/10.1001/jama neurol.2017.4198.

7. Raghavan NS, Dumitrescu L, Mormino E, Mahoney ER, Lee AJ, Gao Y, et al. Association between common variants in RBFOX1, an RNA-binding protein, and brain amyloidosis in early and preclinical Alzheimer disease. JAMA Neurol. 2020;77(10):1288-98. https://doi.org/10.1001/jamaneurol.2020.1760.

8. Li J, Zhang Q, Chen F, et al. Genetic interactions explain variance in cingulate amyloid burden: an AV-45 PET genome-wide association and interaction study in the ADNI cohort. Biomed Res Int. 2015;2015:647389.

9. Ramanan VK, Risacher SL, Nho K, Kim S, Shen L, McDonald BC, et al. GWAS of longitudinal amyloid accumulation on 18F-florbetapir PET in Alzheimer's disease implicates microglial activation gene IL1RAP. Brain. 2015;138(10): 3076-88. https://doi.org/10.1093/brain/awv231.

10. Ramanan VK, Risacher SL, Nho K, et al. APOE and BCHE as modulators of cerebral amyloid deposition: a florbetapir PET genome-wide association study. Mol Psychiatry. 2014;19(3):351-7. https://doi.org/10.1038/mp.2013.19.

11. Yan Q, Nho K, Del-Aguila JL, et al. Genome-wide association study of brain amyloid deposition as measured by Pittsburgh Compound-B (PiB)-PET imaging. Mol Psychiatry. 2021;26(1):309-21.

12. Adeyemo A, Rotimi C. Genetic variants associated with complex human diseases show wide variation across multiple populations. Public Health Genomics. 2010;13(2):72-9. https://doi.org/10.1159/000218711.

13. Li YR, Keating BJ. Trans-ethnic genome-wide association studies: advantages and challenges of mapping in diverse populations. Genome Med. 2014; 6(10):91. https://doi.org/10.1186/s13073-014-0091-5.

14. Carlson CS, Matise TC, North KE, Haiman CA, Fesinmeyer MD, Buyske S, et al. Generalization and dilution of association results from European GWAS in populations of non-European ancestry: the PAGE study. PLoS Biol. 2013; 11(9):e1001661. https://doi.org/10.1371/journal.pbio.1001661.

15. Kunkle BW, Schmidt M, Klein H-U, et al. Novel Alzheimer disease risk loci and pathways in African American individuals using the African genome resources panel: a meta-analysis. JAMA Neurol. 2021;78(1):102-13.

16. Popejoy AB, Fullerton SM. Genomics is failing on diversity. Nature. 2016; 538(7624):161-4. https://doi.org/10.1038/538161a.

17. Petrovski S, Goldstein DB. Unequal representation of genetic variation across ancestry groups creates healthcare inequality in the application of precision medicine. Genome Biol. 2016;17:1-3.

18. Martin AR, Kanai M, Kamatani Y, Okada Y, Neale BM, Daly MJ. Clinical use of current polygenic risk scores may exacerbate health disparities. Nat Genet. 2019;51(4):584-91. https://doi.org/10.1038/s41588-019-0379-X.

19. Hwang J, Jeong JH, Yoon SJ, Park K, Kim EJ, Yoon B, et al. Clinical and biomarker characteristics according to clinical spectrum of Alzheimer's 
disease (AD) in the validation cohort of Korean Brain Aging Study for the Early Diagnosis and Prediction of AD. J Clin Med. 2019;8(3):341. https://doi. org/10.3390/jcm8030341.

20. Lee DY, Lee KU, Lee JH, Kim KW, Jhoo JH, Kim SY, et al. A normative study of the CERAD neuropsychological assessment battery in the Korean elderly. J Int Neuropsychol Soc. 2004;10(1):72-81. https://doi.org/10.1017/S135561 7704101094

21. McKhann GM, Knopman DS, Chertkow H, et al. The diagnosis of dementia due to Alzheimer's disease: recommendations from the National Institute on Aging-Alzheimer's Association workgroups on diagnostic guidelines for Alzheimer's disease. Alzheimers Dement. 2011;7(3):263-9. https://doi.org/1 0.1016/j.jalz.2011.03.005.

22. Kang SH, Park YH, Lee D, Kim JP, Chin J, Ahn Y, et al. The cortical neuroanatomy related to specific neuropsychological deficits in Alzheimer's continuum. Dement Neurocogn Disord. 2019;18(3):77-95. https://doi.org/1 0.12779/dnd.2019.18.3.77.

23. Petersen RC. Mild cognitive impairment. N Engl J Med. 2011;364(23):222734. https://doi.org/10.1056/NEJMcp0910237.

24. Purcell S, Neale B, Todd-Brown K, Thomas L, Ferreira MAR, Bender D, et al, PLINK: a tool set for whole-genome association and population-based linkage analyses. Am J Hum Genet. 2007;81(3):559-75. https://doi.org/10.1 086/519795.

25. Fuchsberger C, Abecasis GR, Hinds DA. minimac2: faster genotype imputation. Bioinformatics. 2015;31(5):782-4. https://doi.org/10.1093/ bioinformatics/btu704

26. Howie B, Fuchsberger C, Stephens M, Marchini J, Abecasis GR. Fast and accurate genotype imputation in genome-wide association studies through pre-phasing. Nat Genet. 2012;44(8):955-9. https://doi.org/10.1038/ng.2354.

27. Thurfjell L, Lilja J, Lundqvist R, Buckley C, Smith A, Vandenberghe R, et al. Automated quantification of 18F-flutemetamol PET activity for categorizing scans as negative or positive for brain amyloid: concordance with visual image reads. J Nucl Med. 2014;55(10):1623-8. https://doi.org/10.2967/ jnumed.114.142109.

28. Curtis C, Gamez JE, Singh U, Sadowsky CH, Villena T, Sabbagh MN, et al. Phase 3 trial of flutemetamol labeled with radioactive fluorine 18 imaging and neuritic plaque density. JAMA Neurol. 2015;72(3):287-94. https://doi. org/10.1001/jamaneurol.2014.4144.

29. Stranger BE, Stahl EA, Raj T. Progress and promise of genome-wide association studies for human complex trait genetics. Genetics. 2011;187(2): 367-83. https://doi.org/10.1534/genetics.110.120907.

30. Nishino J, Ochi H, Kochi Y, Tsunoda T, Matsui S. Sample size for successful genome-wide association study of major depressive disorder. Front Genet. 2018;9:227. https://doi.org/10.3389/fgene.2018.00227.

31. Stein MB, McCarthy MJ, Chen C-Y, et al. Genome-wide analysis of insomnia disorder. Mol Psychiatry. 2018;23(11):2238-50. https://doi.org/10.1038/s413 80-018-0033-5.

32. Devlin B, Roeder K, Wasserman L. Genomic control, a new approach to genetic-based association studies. Theor Popul Biol. 2001;60(3):155-66. https://doi.org/10.1006/tpbi.2001.1542

33. Vandenberghe R, Van Laere K, Ivanoiu A, et al. 18F-flutemetamol amyloid imaging in Alzheimer disease and mild cognitive impairment: a phase 2 trial. Ann Neurol. 2010;68(3):319-29. https://doi.org/10.1002/ana.22068.

34. Bullich S, Seibyl J, Catafau AM, Jovalekic A, Koglin N, Barthel H, et al. Optimized classification of 18F-Florbetaben PET scans as positive and negative using an SUVR quantitative approach and comparison to visual assessment. Neuroimage Clin. 2017;15:325-32. https://doi.org/10.1016/j.nicl.2 017.04 .025$.

35. Consortium GP. A global reference for human genetic variation. Nature. 2015;526(7571):68-74. https://doi.org/10.1038/nature15393.

36. Carithers $L$, Moore HM. The genotype-tissue expression (GTEx) project. Biopreserv Biobank. 2015;13(5):307-8. https://doi.org/10.1089/bio.2015.29031. hmm.

37. Palmqvist $S$, Schöll $M$, Strandberg $O$, et al. Earliest accumulation of $\beta$ amyloid occurs within the default-mode network and concurrently affects brain connectivity. Nat Commun. 2017:8:1-13.

38. Jack CR Jr, Bennett DA, Blennow K, Carrillo MC, Dunn B, Haeberlein SB, et al. NIA-AA research framework: toward a biological definition of Alzheimer's disease. Alzheimers Dement. 2018;14(4):535-62. https://doi.org/10.1016/j.ja Iz.2018.02.018

39. Fargo KN, Carrillo MC, Weiner MW, Potter WZ, Khachaturian Z. The crisis in recruitment for clinical trials in Alzheimer's and dementia: an action plan for solutions. Alzheimers Dement. 2016;12(11):1113-5. https://doi.org/10.1016/j. jalz.2016.10.001.

40. McGilvray ID, Lu Z, Wei AC, et al. Murine hepatitis virus strain 3 induces the macrophage prothrombinase fgl-2 through p38 mitogen-activated protein kinase activation. J Biol Chem. 1998;273(48):32222-9. https://doi.org/10.1 074/jbc.273.48.32222.

41. Rabizadeh E, Cherny I, Lederfein D, Sherman S, Binkovsky N, Rosenblat Y, et al. The cell-membrane prothrombinase, fibrinogen-like protein 2 , promotes angiogenesis and tumor development. Thromb Res. 2015;136(1): 118-24. https://doi.org/10.1016/j.thromres.2014.11.023.

42. Chan CW, Kay LS, Khadaroo RG, et al. Soluble fibrinogen-like protein 2/ fibroleukin exhibits immunosuppressive properties: suppressing $T$ cell proliferation and inhibiting maturation of bone marrow-derived dendritic cells. J Immunol. 2003;170(8):4036-44. https://doi.org/10.4049/jimmunol.170. 8.4036.

43. Yan J, Kong L-Y, Hu J, et al. FGL2 as a multimodality regulator of tumormediated immune suppression and therapeutic target in gliomas. J Natl Cancer Inst. 2015;107:djv137.

44. Yan J, Zhao Q, Gabrusiewicz K, et al. FGL2 promotes tumor progression in the CNS by suppressing CD103+ dendritic cell differentiation. Nat Commun. 2019;10:1-15.

45. Walker DG, Link J, Lue LF, Dalsing-Hernandez JE, Boyes BE. Gene expression changes by amyloid $\beta$ peptide-stimulated human postmortem brain microglia identify activation of multiple inflammatory processes. J Leukoc Biol. 2006;79(3):596-610. https://doi.org/10.1189/jlb.0705377.

46. Taguchi K, Yamagata HD, Zhong W, Kamino K, Akatsu H, Hata R, et al. Identification of hippocampus-related candidate genes for Alzheimer's disease. Ann Neurol. 2005;57(4):585-8. https://doi.org/10.1002/ana.20433.

47. Farrer LA, Cupples LA, Haines JL, et al. Effects of age, sex, and ethnicity on the association between apolipoprotein E genotype and Alzheimer disease: a meta-analysis. JAMA. 1997;278(16):1349-56. https://doi.org/10.1001/jama.1 997.03550160069041.

48. Wu P, Li H-L, Liu Z-J, Tao QQ, Xu M, Guo QH, et al. Associations between apolipoprotein E gene polymorphisms and Alzheimer's disease risk in a large Chinese Han population. Clin Interv Aging. 2015;10:371-8. https://doi. org/10.2147/CIA.S73396

49. Sabri O, Sabbagh MN, Seibyl J, Barthel H, Akatsu H, Ouchi $Y$, et al. Florbetaben PET imaging to detect amyloid beta plaques in Alzheimer's disease: phase 3 study. Alzheimers Dement. 2015;11(8):964-74. https://doi. org/10.1016/j.jalz.2015.02.004

50. Salloway S, Gamez JE, Singh U, Sadowsky CH, Villena T, Sabbagh MN, et al. Performance of [18F] flutemetamol amyloid imaging against the neuritic plaque component of CERAD and the current (2012) NIA-AA recommendations for the neuropathologic diagnosis of Alzheimer's disease. Alzheimers Dement. 2017;9(1):25-34. https://doi.org/10.1016/j.da dm.2017.06.001.

\section{Publisher's Note}

Springer Nature remains neutral with regard to jurisdictional claims in published maps and institutional affiliations.
Ready to submit your research? Choose BMC and benefit from:

- fast, convenient online submission

- thorough peer review by experienced researchers in your field

- rapid publication on acceptance

- support for research data, including large and complex data types

- gold Open Access which fosters wider collaboration and increased citations

- maximum visibility for your research: over $100 \mathrm{M}$ website views per year

At $\mathrm{BMC}$, research is always in progress.

Learn more biomedcentral.com/submissions 